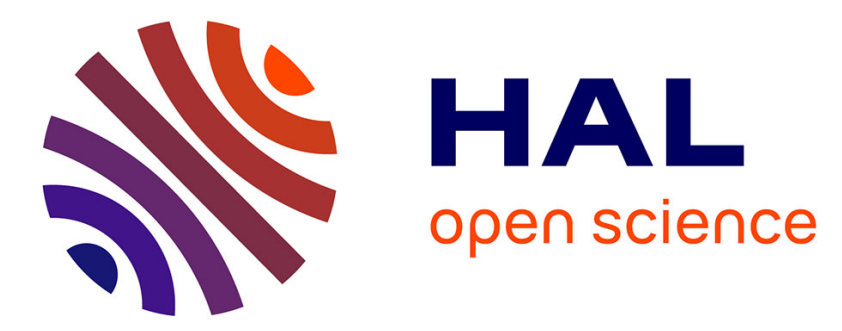

\title{
Non-Additive Approach for Omnidirectional Image Gradient Estimation
}

Florence Jacquey, Frédéric Comby, Olivier Strauss

\section{To cite this version:}

Florence Jacquey, Frédéric Comby, Olivier Strauss. Non-Additive Approach for Omnidirectional Image Gradient Estimation. ICCV'07: International Conference on Computer Vision OMNIVIS'07: Workshop on Omnidirectional Vision, Camera Networks and Non-classical Cameras, Oct 2007, Rio de Janeiro, Brésil, pp.1-4. lirmm-00194216

\section{HAL Id: lirmm-00194216 https://hal-lirmm.ccsd.cnrs.fr/lirmm-00194216}

Submitted on 6 Dec 2007

HAL is a multi-disciplinary open access archive for the deposit and dissemination of scientific research documents, whether they are published or not. The documents may come from teaching and research institutions in France or abroad, or from public or private research centers.
L'archive ouverte pluridisciplinaire HAL, est destinée au dépôt et à la diffusion de documents scientifiques de niveau recherche, publiés ou non, émanant des établissements d'enseignement et de recherche français ou étrangers, des laboratoires publics ou privés. 


\title{
Non-additive Approach for Omnidirectional Image Gradient Estimation
}

\author{
Florence JACQUEY, Frédéric COMBY, Olivier STRAUSS \\ LIRMM, Univ. Montpellier 2, CNRS , 161 rue Ada, 34392 Montpellier Cedex 5 France \\ \{jacquey, comby, strauss\}@lirmm.fr
}

\begin{abstract}
The way catadioptric images are acquired implies that they present radial distortions. Therefore, classical processing may not be suitable. This statement will be illustrated by considering edge detection matter. Classical edge detectors usually consist in three steps : gradient computation, maximization and thresholding. The two lasts steps use pixels neighborhood concept. On the opposite of perspective images where pixel neighborhood is intuitive, catadioptric images present radial resolution changes. Then, the size and shape of pixel neighborhood have to be depending on pixel location. This article presents a new gradient estimation approach based on non-additive kernels. This technique is adapted to catadioptric images and also provides a natural threshold discarding the arbitrary thresholding step.
\end{abstract}

\section{Introduction}

Omnidirectional vision aims to enhance the perspective cameras field of view. Many applications, such as mobile robotics, video-conference, virtual reality representations, have found an interest in this approach. Among all ways to enlarge the field of view, the catadioptric approach is one of the most frequently used. These sensors acquire $360^{\circ}$ images by combining a perspective camera with a revolution mirror. However, the mirror geometry provides important radial distortions on the image. Moreover, the image sampling combined with the distorsion brought by the mirror leads to a non-uniform resolution all over the image (the image resolution is lower at the center than at the periphery). Such phenomena complicate omnidirectional image processing.

This article focuses on the gradient-based edge detection on omnidirectional images. Edge detection is a preliminary crucial stage for many applications. Edges are characterized by the discontinuities in the intensity function. Discrete image processing algorithms often derive from continuous signal processing. To ensure a kind of continuity on discrete images, an interpolation is needed. This interpolation is performed by convolving kernels with the image. Kernels define a weighted neighborhood, providing an interplay between continuous and discrete domains of each sampled location. This neighborhood reduces the effect of random noise by providing regularization in the interpolation process. A pixel value is computed by a linear combination of the grey levels values of this pixel and its neighborhood.

The gradient-based approach consists in extracting the local maxima of the intensity function first derivative. Thus, the gradient norm at pixel $P_{i, j}$ is compared with the two neighbors gradient norms in the gradient direction. This neighbors (noted $P_{A}$ and $P_{B}$ ) are supposed to be at a unit distance on both sides of $P_{i, j}$. An edge point is detected if the gradient value is maximum along this line in $P_{i, j}$.

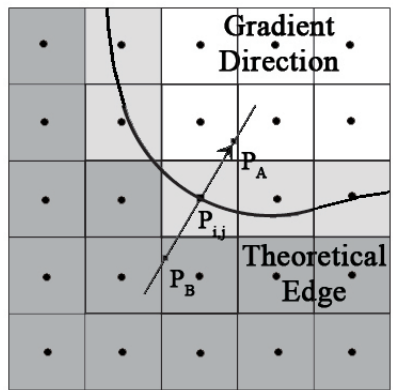

(a) perspective image

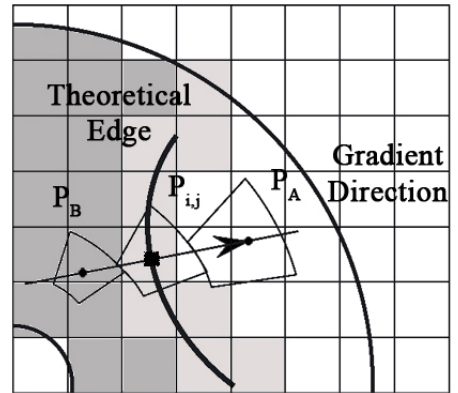

(b) omnidirectional image
FIG. 1. Gradient local extrema extraction.

For perspective images, where pixels constitute a regular Cartesian grid of the projected scene, the neighborhood concept is rather intuitive. Each pixel neighborhood is defined for a given direction and the gradient norm is computed by linear interpolation. On Figure 1(a), the pixel $P_{i, j}$ is an edge point if $\left\|\nabla_{I}\left(P_{i, j}\right)\right\|>\left\|\nabla_{I}\left(P_{A}\right)\right\|$ and $\left\|\nabla_{I}\left(P_{i, j}\right)\right\|>\left\|\nabla_{I}\left(P_{B}\right)\right\|$ where $\left\|\nabla_{I}(P)\right\|$ is the gradient norm of the image $I$ at location $P$.

On catadioptric images, the neighborhood topology is modified by the mirror geometry. The definition of $P_{A}$ and $P_{B}$ is less trivial. In fact, the projection of the 3D scene on mirror and retina concentrates the information on the center of the image : the resolution is not radially invariant. Thus, the definition of two points distant of one unit from $P_{i, j}$ 
is meaningless on the image. It is necessary to find these two points in a representation space where distances are homogeneous whatever $P_{i, j}$ location may be on the image. A solution to define the $P_{i, j}$ neighbors in the gradient direction consists in using the projection models described in [15] via the cylinder. Figure 1(b) illustrates the computation of the two "pixels" $P_{A}$ and $P_{B}$ using the cylindrical projective model. However, the computing time of the projective approach is very expensive : a pre-computation is quite impossible because the gradient direction can vary for each pixel.

Many solutions have been proposed to process omnidirectional images in the literature. Let's remind that catadioptric sensors satisfying the single view point constraint [16], allow the generation of geometrically correct perspective images from one omnidirectional image. A first intuitive approach consists in reconstructing the image in another space (perspective, cylindrical, spherical, etc.) to process it. This approach involves interpolations and smoothing that modify the image grey levels. The mirror geometry plays a central role in the omnidirectional images formation and must be carefully taken into account in image processing. In [9] the partial differential equations are used to cope with geometrical constraints and adapt finite differences filters. Some authors have redefined these operators using the unified projection model [2]. In [12], the omnidirectional image is projected onto a virtual sphere to define the gradient. In [17], the authors develop convolution tools directly on the sphere. Spherical images are also used in [10] to compute the correlation function. Another solution is to project, on each pixel, a kernel whose geometry is defined in a more suitable space using the system calibration parameters. In [1], the pixel neighborhood is defined with the unified projection model to apply a Markov random fields. The authors of [18] deals with the interest points mapping problem between two catadioptric images : the proposed solution consists in using the neighborhood geometry whose size depends on the image position. A similar method is presented in [15] : the neighborhood is geometrically defined on a cylinder and projected on the omnidirectional image. Fuzzy morphological kernels are used to deals with the imprecisions brought by the image sampling and neighborhood localization. In [8], this fuzzy model is used to adapt convolution masks to omnidirectional images. In medical imaging [14], an image processing method is developped directly on echographic images, which are not really omnidirectional images, but still present distortions due to the image acquisition process.

In this article, we introduce a non-additive approach for gradient-based edge detection adapted to the omnidirectional images geometry. This approach avoids the maximization and threshold stages by providing a natural threshold. This natural threshold provides robustness against uniform and non-uniformly noise.

\section{Summative and Non-Summative Kernels}

This chapter briefly explains the basic notions on kernels operations (summative and non-summative). These techniques are used in image processing to estimate the continuous signal knowing the discrete one.

\subsection{Summative Kernels}

Summative kernels are $\mathbb{R}$-valued functions $\kappa$ defined on a domain $\Omega$, satisfying the summative normalization property :

$$
\int_{\Omega} \kappa(\omega) d \omega=1 .
$$

In image processing they are usually positive, centered, bounded, uni-modal and symmetric. Positivity implies that they can be seen as probability density functions, associated to a probability measure $P_{\kappa}: \forall A \subseteq \Omega, P_{\kappa}(A)=$ $\int_{A} \kappa(\omega) d \omega$.

Estimation of a signal $S$ convolved with a summative or probabilistic neighborhood $\kappa$ is the expectation of this signal according to $\kappa$ :

$$
\mathbb{E}_{\kappa}(S)=\int_{\Omega} S d P_{\kappa}=\int_{\Omega} S(\omega) \kappa(\omega) d \omega .
$$

\subsection{Non-Summative Kernels}

Non-summative kernels are $[0,1]$-valued functions $\pi$ defined on a domain $\Omega$, satisfying the maximitive normalization property :

$$
\max _{\omega \in \Omega} \pi(\omega)=1 .
$$

As a summative kernel is associated to a probability density function, a non summative kernel can be associated to a possibility density function [3] whose mesure $\Pi_{\pi}$ is defined by : $\forall A \in \Omega, \Pi_{\pi}(A)=\sup _{\omega \in A} \pi(\omega)$.

\subsection{Link between Summative and Non-Summative Kernels}

Estimation using kernels provides an interpolation between continuous and discrete. However each summative kernel presents its own properties. How to choose the appropriate one for an estimation?

To be compared, summative kernels have to be normalized to present the same granulosity [13]. In this case, it is possible to choose a non-summative kernel dominating a family of same granulosity summative kernel. This kernel estimation will provide the upper and lower boundaries of all values estimated using the dominated summative kernels.

Dubois and al. [4] have proposed a transformation from a probability distribution to a possibility distribution. Any summative kernel $\kappa$ is associated to a non-summative kernel 
$\pi$ satisfying the domination principle $: \forall A \subseteq \Omega, P_{\kappa}(A) \leq$ $\Pi_{\pi}(A)$. Moreover, they have shown that any summative kernel $\kappa$ bounded on an interval $[-\Delta, \Delta]$ satisfies : $\forall A \subseteq$ $\Omega, P_{\kappa}(A) \leq \Pi_{T}(A)$, where $T$ is the triangular possibility distribution on $[-\Delta, \Delta]$. This means that $T$ dominates any centered symmetric summative kernel. In the following, triangular non-summative kernel will be used as our non summative kernel.

\section{Signal Processing}

\subsection{Non-Summative Kernel Estimation}

The signal sampling introduces an imprecision on the information localization. Using a fuzzy partition $\left(H_{i}\right)_{i \in\{1, \ldots, N\}}$, depicted on Figure 2, to represent the sampling effects allows to take into account this imprecision. However, Equation (2) cannot be used when data are imprecise. Convolution product needs to be replaced by a Choquet integral to transfer the fuzzy partition knowledge to the non-summative kernel $W$. This integral provides two boundaries of the estimation of $W$ value :

$$
\mathbb{C}_{\nu_{W}^{c}}(S) \leq \mathbb{E}_{\kappa}(S) \leq \mathbb{C}_{\nu_{W}}(S)
$$

where $\kappa$ represents any summative kernel dominated by $W$. The Choquet integral is given by :

$$
\mathbb{C}_{\nu_{W}}(S)=\sum_{n=1}^{N} S_{(n)}\left[\nu_{W}\left(A_{(n)}\right)-\nu_{W}\left(A_{(n+1)}\right)\right]
$$

where $S_{n}$ is the real positive value associated with the $n^{\text {th }}$ cell $\mathbf{H}_{\mathbf{n}}$ and (.) indicates a permutation such that $S_{(1)} \leq$ $S_{(2)} \leq \ldots \leq S_{(N)}$. The $A_{(n)}=\left\{\mathbf{H}_{(\mathbf{n})}, \ldots, \mathbf{H}_{(\mathbf{N})}\right\}$ are binary coalitions of cells whose associated values are greater or equal to $S_{(n)}$. Computation of $\mathbb{C}_{\nu_{W}^{c}}(S)$ can be simply

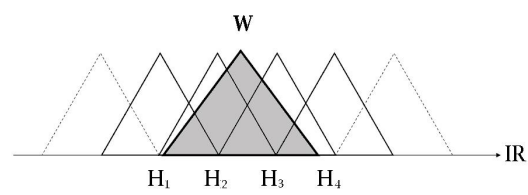

FIG. 2. $\mathbb{R}$ strong triangular partition and fuzzy neighborhood $W$.

achieved by noticing that $\mathbb{C}_{\nu_{W}^{c}}(S)=-\mathbb{C}_{\nu_{W}}(-S)$. We propose to use the pignistic transfer to define the concave capacity used in Equation (5) :

$$
\nu_{W}\left(A_{(n)}\right)=\frac{\left|W \bigcap A_{(n)}\right|}{\left|W \bigcap A_{(1)}\right|} .
$$

where $|X|$ is the set $X$ cardinality (area of $\mathrm{X}$ ).

\subsection{Derivation with Non-Summative Kernel}

Estimation of the continuous signal $s$ derivative results from the convolution of discrete signal $S$ with the derivative summative kernel $d \kappa$. Most of usual approaches lead to a derivative kernel obtained by subtracting two usual kernels $\eta^{+}$and $\eta^{-}$[7] :

$$
d \kappa(x)=\eta^{+}\left(x-\epsilon_{x}\right)-\eta^{-}\left(x+\epsilon_{x}\right) .
$$

Convolving signal $S$ with the derivative kernel given by expression (7) is equivalent to add the convolution of $S$ with the kernel $\eta^{+}$and the convolution of the opposite of $S$ with kernel $\eta^{-}$:

$$
\begin{aligned}
D S(x) & =\left(\eta^{+}\left(x+\epsilon_{x}\right)-\eta^{-}\left(x-\epsilon_{x}\right)\right) * S(x) \\
& =\eta^{+}\left(x+\epsilon_{x}\right) * S(x)+\eta^{-}\left(x-\epsilon_{x}\right) *(-S(x)) .
\end{aligned}
$$

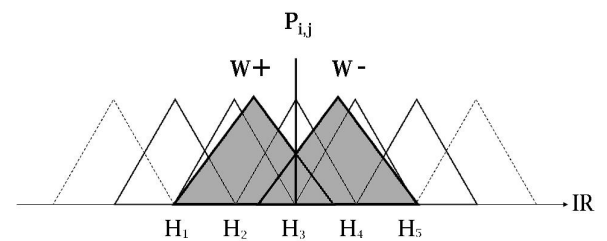

FIG. 3. Derivative kernel (subtraction of two usual kernels).

Let's define $\kappa^{+}(x)=\eta^{+}\left(x+\epsilon_{x}\right)$ and $\kappa^{-}(x)=\eta^{-}\left(x-\epsilon_{x}\right)$. The estimation of $S$ derivative at location $x(D S(x))$ is :

$$
\begin{gathered}
D S(x)=\mathbb{E}_{\kappa^{+}}(S)+\mathbb{E}_{\kappa^{-}}(-S) \\
\text { with }\left\{\begin{array}{l}
\mathbb{E}_{\kappa^{+}}(S) \in\left[-\mathbb{C}_{\nu_{W}^{+}}(-S), \mathbb{C}_{\nu_{W}^{+}}(S)\right] \\
\mathbb{E}_{\kappa^{-}}(-S) \in\left[-\mathbb{C}_{\nu_{W}^{-}}(S), \mathbb{C}_{\nu_{W}^{-}}(-S)\right]
\end{array}\right.
\end{gathered}
$$

If $\kappa^{+}$(resp. $\kappa^{-}$) is a bounded, symmetric and positive kernel, then the triangular possibility distribution can be used to ensure its domination (see Section 2). Expression (9) gives an imprecise estimation of the discrete signal derivative :

$$
\begin{aligned}
& {[\underline{D S}, \overline{D S}]} \\
& =\left[-\mathbb{C}_{\nu_{W}^{+}}(-S), \mathbb{C}_{\nu_{W}^{+}}(S)\right]+\left[-\mathbb{C}_{\nu_{W}^{-}}(S), \mathbb{C}_{\nu_{W}^{-}}(-S)\right] \\
& =\left[-\mathbb{C}_{\nu_{W}^{+}}(-S)-\mathbb{C}_{\nu_{W}^{-}}(S), \mathbb{C}_{\nu_{W}^{+}}(S)+\mathbb{C}_{\nu_{W}^{-}}(-S)\right] .
\end{aligned}
$$

If $\nu_{W}$ is a concave capacity, this interval contains all the derivative possible values.

\subsection{Edge Detection with non-summative kernel ba- sed gradient}

Images are 2D discrete signals, then the grey level localization is imprecise. Let $P_{i, j}$ be the pixel located at $(i, j)$ on the image. Its grey level localization is unknown within the 2D interval $\left[i-\Delta_{h}, i+\Delta_{h}\right] \times\left[j-\Delta_{v}, j+\Delta_{v}\right]$, where $\Delta_{h}$ 
(resp. $\Delta_{v}$ ) is half the width of the horizontal (resp. vertical) sampling. This imprecision is due to the spatial sampling and can be modeled by a fuzzy partition [15]. Each pixel of the image is considered as a bi-dimensional imprecise quantity. The 1-D triangular fuzzy numbers presented in Section 2 are extended in 2D pyramidal fuzzy numbers (Figure 4(a)) using the t-norm min. They provide a strong fuzzy partition of the image.

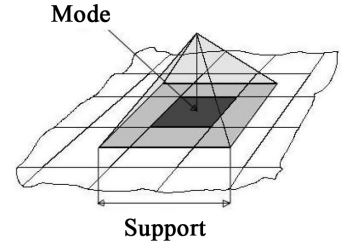

(a) Fuzzy pixel

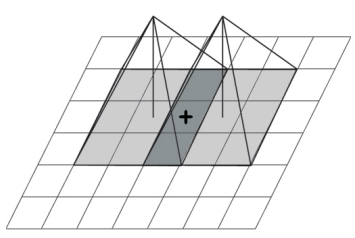

(b) 2D non-summative kernels
FIG. 4. Fuzzy representation.

Non-summative kernels are also extended in 2D to compute an imprecise gradient estimation. Let $\kappa(x, y)=$ $\kappa_{x}(x) \kappa_{y}(y)$ be a separable kernel. The $2 \mathrm{D}$ extension of expression (7) along the $x$-axis is given by :

$$
\begin{aligned}
\frac{\delta}{\delta x}(\kappa(x, y)) & =\frac{\delta}{\delta x}\left(\kappa_{x}(x) \kappa_{y}(y)\right)=\frac{\delta}{\delta x}\left(\kappa_{x}(x)\right) \kappa_{y}(y) \\
& =\left(\eta^{+}\left(x+\epsilon_{x}\right)-\eta^{-}\left(x-\epsilon_{x}\right)\right) \kappa_{y}(y) .
\end{aligned}
$$

Let $\kappa_{x}^{+}=\eta^{+}\left(x+\epsilon_{x}\right) \kappa_{y}(y)$ and $\kappa_{x}^{-}=\eta^{-}\left(x-\epsilon_{x}\right) \kappa_{y}(y)$ be two summative kernels. An estimation of the x-component 2D signal $S\left(G S_{x}\right)$ is given by the extension of Equation (9) :

$$
G S_{x}=\mathbb{E}_{\kappa_{x}^{+}}(S)+\mathbb{E}_{\kappa_{x}^{-}}(-S) .
$$

As $\mathbb{E}_{\kappa_{x}^{+}}(S)$ and $\left.\mathbb{E}_{\kappa_{x}^{-}}(-S)\right)$ are imprecise quantities, $G S_{x}$ is also imprecise. The triangular kernel in Equation (5) is generalized into a pyramidal one.

As different kind of noise can produce spurious edges, a thresholding step is necessary to keep relevant edges. Our method produces an interval for the gradient estimation and we can assume that if $(0,0) \in\left[\underline{G S_{x}}, \overline{G S_{x}}\right] \times\left[\underline{G S_{y}}, \overline{G S_{y}}\right]$, the extremum value can be discarded.

\subsection{Adaptation to omnidirectional images}

The operator defined in Section 3.3 is regularly sampled. It is relevant to perspective images with a Cartesian partition, but as seen in Section 1, omnidirectional images present radial distorsions and resolution variations. As pointed out in [12], it is crucial that data are kept in their original space. Thus, if data are unchanged the operator needs to be modified. Instead of projecting pixels values on another support, the kernel will be geometrically defined on a virtual space and projected on the omnidirectional image. To remain independent of the mirror, the unifying projection theory of Geyer and Daniilidis is used. The projective space used to define the kernels is the surrounding cylinder. Indeed, even if it presents some distorsions, this representation is very easy to sample and quasi equivalent to a perspective image when the kernel size remains small. Therefore, the approximation brought by the cylinder, with respect to the perspective plane, is almost negligible for a small kernel (the localization error on our projected kernel is about $5.10^{-5}$ pixels in the worst case). Recall that the image is not mapped on the cylinder; it is only a projective space, where kernels can be easily defined.

Our non-summative gradient operator is adapted to the omnidirectional image geometry with the following algorithm:

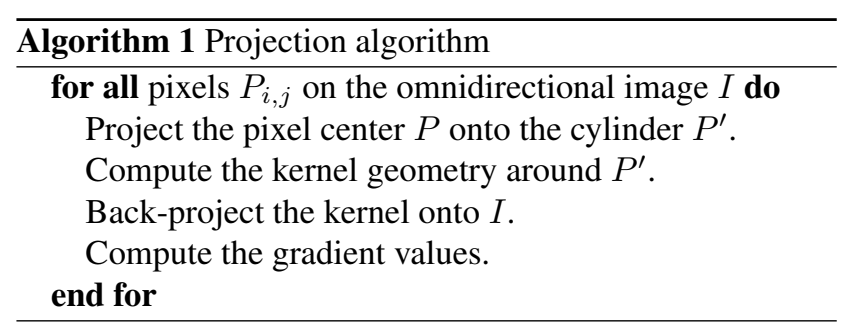

Figure 5 illustrates the projection of a pixel center on the cylinder. Our fuzzy derivative kernel is defined around this projection and back-projected on the panoramic image. The kernels size depends on the size of the sought after edges. The gradient value of the filtered pixel is computed with respect to the projected kernel.

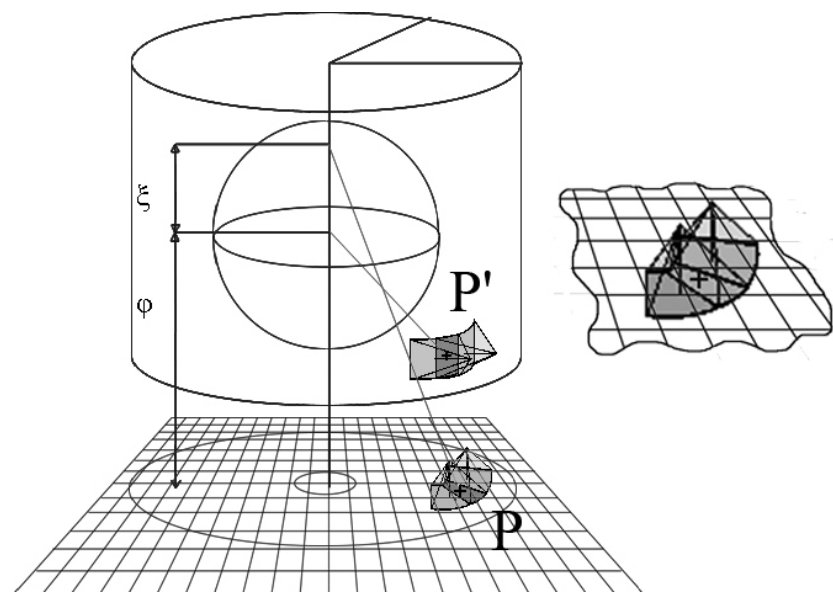

FIG. 5. Kernel projection on the omnidirectional image and detail on the omnidirectional image.

The derivative kernel covers many pixels of the panoramic image (Figure 5 detail). The gradient interval, described by (11), associated to each projected kernel, is computed by a Choquet integral (5). 


\section{Experiments}

Our approach (called Gradient on the figures) is compared with the Prewitt approach, its omnidirectional adaptation presented in [8] (called Adaptation on the figures) and the optimal approaches of Canny-Deriche [5] and ShenCastan [11] on an artificial image. Real image experiments are also presented.

\subsection{Quantitative Estimation of Noise Sensibility}

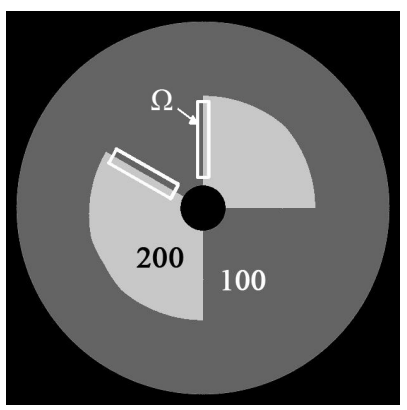

(a) Artificial image

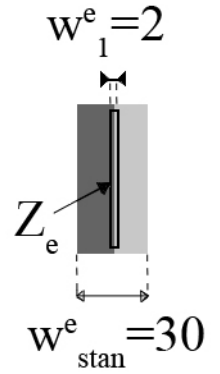

(b) Zoom on $\Omega$.
FIG. 6. Synthetic image used for robustness tests.

In this section, we quantitatively evaluate the noise sensibility of our approach on an artificial omnidirectional image depicted in Figure 6(a). This image is composed by two regions whose grey levels are 100 and 200. On this image, we have the total control of all parameters (camera, mirror, etc). This image has been corrupted with different kinds of noise to compare the robustness of the edge detectors. The parameter $P_{1}$, defined by Fram and Deutsch in [6], measures the sensitivity of the detector in presence of noise. $P_{1}=1$ if the edge detection is optimal.

$$
P_{1}=\frac{n_{\text {sig }}^{e}}{n_{\text {sig }}^{e}+\left(n_{\text {noise }}^{e}+n^{0}\right) \frac{n_{\text {in }}}{f n_{\text {tot }}}}
$$

where $n_{\text {sig }}^{e}=\frac{n^{e}-n_{\text {noise }}^{e}}{1-\frac{n_{\text {noise }}^{e}}{n_{\text {in }}}}$ and $n_{\text {noise }}^{e}=n^{0} \frac{n_{\text {in }}}{n_{\text {out }}}$. Figure 6(b) is a zoom on $\Omega$ (the white squared image region on Figure 6(a)). $\Omega$ represents the considered region for the $P_{1}$ estimation. This region is composed by $w_{\text {stan }}^{e}=30$ columns here. Let $Z_{e}$ be the edge zone in $\Omega$. $Z_{e}$ contains $w_{1}^{e}=2$ columns here. $n_{\text {tot }}$ is the total number of points flagged as edge points by the edge detector, $n_{i n}$ the part of $n_{t o t}$ inside $Z_{e}$ and $n_{\text {out }}$ the part outside $Z_{e}$ such as $n_{\text {tot }}=n_{\text {in }}+n_{\text {out }}$. $n^{0}$ and $n^{e}$ are respectively the numbers of points flagged as edge points outside and inside $Z_{e}$ after thresholding. Finally, let $\overline{Z_{e}}=\Omega-Z_{e}$, parameter $f$ is used to normalize the edge detector output such as the points proportion between $Z_{e}$ and $\overline{Z_{e}}$ is conserved : $f=\frac{w_{1}^{e}}{w_{\text {stan }}^{e}}$.

The first experiment consists in adding a Gaussian noise with an increasing variance $\sigma$ to the image. First, edges are detected without maximization and threshold. Figure 7(a) shows that all approaches except ours fail when $\sigma>2$. Then, for each method, the gradient has been maximized and thresholded, to provide the best result within the $P_{1}$ criterion. Figure 7(b) shows that the Prewitt filter and its adaptation are the most sensible to noise. Our approach gives good results : optimal edges are detected while $\sigma<$ 45. When $\sigma>45$, all gradient estimations are discarded as being null. Only the Canny-Deriche approach can detect an optimal edge when $\sigma<70$.

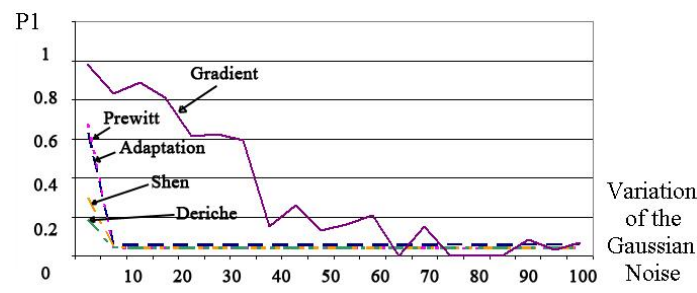

(a) without maximization and threshold

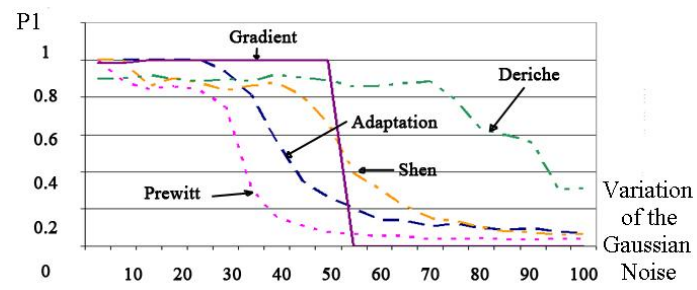

(b) with maximization and threshold

FIG. 7. Noise sensibility of the edge detector.

Avoiding thresholding is highly valuable when images are computed with a non-uniform noise. For example, tomographic images present noise following a Poisson noise distribution. Some results are presented on Table 1. The fuzzy approach is 8 times less sensitive than the Prewitt approach and 5 times less than the Canny-Deriche and Shen-Castan approaches.

\begin{tabular}{|l|l|l|l|l|}
\hline Approche & Prewitt & Deriche & Shen & Fuzzy \\
\hline$P_{1}$ & 0.089676 & 0.14975 & 0.141 & 0.69881 \\
\hline \multicolumn{4}{|c}{ TAB. } \\
\hline
\end{tabular}

\subsection{Experiments on real images}

This section presents some results on a real image depicted 8(a) to illustrate the good performances of our approach. The mirror is hyperbolic and the sensor is calibrated. Our image includes a grid made of black parallel lines. Half of the lines are thin, the other half are thick. Due to distortions brought by the projection onto the mirror, the black lines seems to get thinner at the image center. Figure 8(b) shows the edge detection without post-processing stage (no gradient maximization nor threshold). High grey level values (white) stand for high gradient values. 
A good edge detector is able to find for each image line a double edge from the periphery to the center. We can observe that edges are correctly identified on the omnidirectional image. The two edges are well highlighted and remain separated from the periphery to the image center.

Optimal gradient operators (such as Canny-Deriche and Shen-Castan) will also provide a good edge detection on omnidirectional image. Nevertheless, their behavior will remain the same all over the image as if it was a perspective one. They discard the resolution variations. Conversely, our approach keeps the same behavior on the cylindrical projective space. This space, locally close to a perspective image, allows to detect edges in a coherent manner with respect to the real scene. Indeed, the edge thickness depends on the radial position on the omnidirectional image.

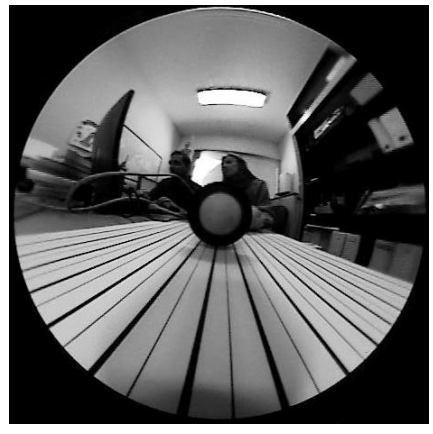

(a) Original image

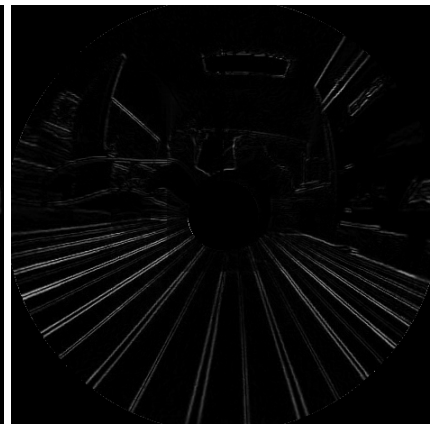

(b) Edges detected
FIG. 8. Edge detection on real image.

\section{Conclusion}

In this article, a new non-additive approach for gradientbased edge detection on omnidirectional images is presented. This approach uses a Choquet integral to estimate an imprecise value of the image gradient (an upper and lower boundary of the gradient). A simple rule applied to this interval allows to avoid maximization and thresholding phases in the edge detection process. This is highly valuable as an omnidirectional image processing; these two phases being relatively complex as they depend on the location on the image. Non-summative kernels are adapted to the image geometry by a projection on a virtual surrounding cylinder. This allows to work directly in the image space instead of projecting the image and add noise. The tests give good results on artificial and real images. Further works will be carried out to quantitatively evaluate our algorithm performances applied to interest points detection.

\section{Références}

[1] P. Vasseur C. Demonceaux. Markov random fields for catadioptric image processing. Pattern Recogn. Lett., 27(16) :1957-1967, 2006.
[2] K. Daniilidis C. Geyer. Catadioptric projective geometry. International Journal of Computer Vision, 43 :223-243, 2001.

[3] H. Prade D. Dubois. Possibility Theory An Approach to Computerized Processing of Uncertainty. Plenum Press, 1988.

[4] L. Foulloy G. Mauris D. Dubois, H. Prade. Probabilitypossibility transformations, triangular fuzzy sets, and probabilistic inequalities. Reliable Computing, 10 :273-297, 2004.

[5] R. Deriche. Fast algorithms for low-level vision. IEEE Trans. Pattern Anal. Mach. Intell., 12(1) :78-87, 1990.

[6] J.R. Fram E.S. Deutsch. A quantitative study of the orientational bias of some edge detector schemes. IEEE Trans. on Computers, March 1978.

[7] F. Comby O. Strauss F. Jacquey, K. Loquin. Non-additive approach for gradient-based edge detection. ICIP07, 2007.

[8] O. Strauss F. Jacquey, F. Comby. Fuzzy edge detection for omnidirectional images. LFA06, pages 379-386, 2006.

[9] J. Thiran P. Vandergheynst I. Bogdanova, X. Bresson. Scale-space analysis and active contours for omnidirectional images. IEEE Transactions on Image Processing, 2007.

[10] P. Frossard P. Vandergheynst I. Tosic, I. Bogdanova. Multiresolution motion estimation for omnidirectional images. 2005.

[11] S. Castan J. Shen. An optimal linear operator for step edge detection computer vision. Graphics, and Image Processing, $54: 112-133,1992$.

[12] T. Bulow K. Daniilidis, A. Makadia. Image processing in catadioptric planes : Spatiotemporal derivatives and optical flow computation. OMNIVISO2, pages 3-10, 2002.

[13] O. Strauss K. Loquin. About granulosity of sampling kernels. LFA06, pages 387-394, 2006.

[14] I.L. Herlin N. Ayache. A new methodology to analyse time sequences of ultrasound images. 1991.

[15] F. Comby O. Strauss. Fuzzy morphology for omnidirectional images. In ICIP05, volume 2, pages 141-144, Genova, Italy, September 11-14 2005.

[16] S.K. Nayar S. Baker. A theory of single-viewpoint catadioptric image formation. International Journal of Computer Vision, 35(2) :175-196, 1999.

[17] S. Durand E. Mouaddib S. Bigot, D. Kachi. Spherical image denoising and its application to omnidirectional imaging. In VISAPP07, 2007.

[18] T. Pajdla T. Svoboda. Matching in catadioptric images with appropriate windows, and outliers removal. In CAIP01, pages 733-740, London, UK, 2001. Springer-Verlag. 